\title{
Computation of the Euler Number of a Binary Image Composed of Hexagonal Cells
}

\author{
J. H. Sossa-Azuela*1 , E. V. Cuevas-Jiménez ${ }^{2}$, D. Zaldivar-Navarro ${ }^{2}$ \\ ${ }^{1}$ Centro de Investigación en Computación-IPN \\ Av. Juan de Dios Bátiz, esquina con Miguel Othón de Mendizábal \\ Mexico City, C. P. 07738. MEXICO \\ * hsossa@cic.ipn.mx \\ ${ }^{2}$ Centro Universitario de Ciencias Exactas e Ingenierías (CUCEI) \\ Universidad de Guadalajara \\ Av. Revolución 1500 \\ Col. Olímpica C.P. 44430 \\ Guadalajara, Jal, MEXICO
}

\begin{abstract}
Most of the proposals to compute the Euler number of a binary image have been designed to work with images composed of squared cells. Only a few of these methods (in the case of images composed of hexagonal cells) have been reported in literature, although it is known that images composed of hexagonal cells do not suffer from the problems of connectivity frequently found in the case of images composed of squared cells. In this paper, a new way to compute the Euler number $(E)$ of a binary image composed of hexagonal cells is presented. For this, the perimeter $P$ of the isolated regions in the image, their contact perimeter $P_{c}$ and the type $T$ of a cell are used to obtain this important invariant. The proposal can be used alone or in combination with other features to describe any binary planar shape composed of hexagonal pixels for its further recognition.
\end{abstract}

Keywords: Binary image characterization, Perimeter, Contact Perimeter, Euler number or genus, Topological descriptor, Topological invariant.

\section{RESUMEN}

El principal objetivo de este trabajo es el presentar una nueva clase de controlador de tipo retroalimentado, el cual contiene en su estructura una forma polinomial del llamado error de control, el controlador propuesto es aplicado a un quimiostato sulfato-reductor, el cual pudiera ser usado para varios fines biotecnológicos, como la remoción de metales pesados en aguas residuales. El comportamiento a lazo cerrado del quimiostato considerado es teóricamente analizado y se prueba convergencia práctica a la trayectoria óptima seleccionada. La metodología propuesta es aplicada a un modelo cinético de una bacteria sulfato-reductora experimentalmente validado y experimentos numéricos complementarios muestran un comportamiento a lazo cerrado satisfactorio en comparación con otros controladores.

\section{Introduction}

Conventionally, images are digitized and stored as a rectangular array of values. The image is sampled at each point on a two dimensional grid, storing intensity and implicit location information for each sample. It is known that the rectangular grid is by far the most dominant of any grid structure in image processing, although the hexagonal structured pixel grid is considered to be superior to the rectangular grid system in many respects, including neighboring connectivity, [1] and [2].

The use of hexagonal grids to represent digital images has been studied recently, with improvements in charged coupled device (CCD) technology making hexagonal sampling attractive for practical applications and development of new interests in this area [3]. However, some algorithms have been developed to apply processing techniques directly to hexagonal images, [2] and [4]. 
The Euler number of an image is an important feature that can be used to describe the topological structure of that image. It is known that this describing feature is invariant up to several image transformations such as translations, rotations, scale changes, affinities, projections and even some non-linear transformations such as deformation of the shapes contained in the image. Mathematically, the Euler number of a binary image is defined as [5], [6], [7]:

$$
E=N-H
$$

where $N$ is the number of regions of the image (number of connected components of the object) and $\mathrm{H}$ is the number of holes in the image (isolated regions of the image's background). The Euler numbers of the two images shown, for example, in Fig. 1 (a) have, values: 1 (2 regions -1 hole) and 0 (3 regions -3 holes), respectively.

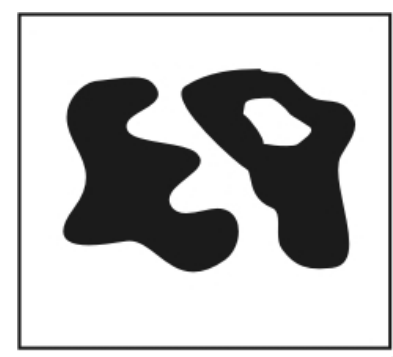

(a)
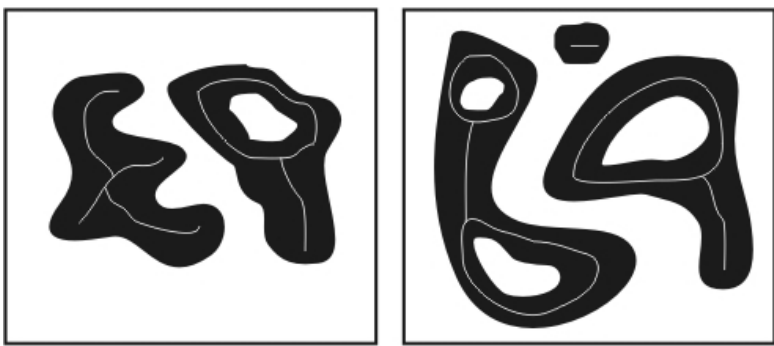

(b)

Fiaure 1. Euler numbers of two simble imaaes.

The Euler number of an image has proven to be an important feature in image analysis and visual inspection applications. The interested reader can make reference, for example, to the work reported in [8].
In [9], the authors propose computing so called fast Euler numbers to automatically threshold a binary image. Their proposal allows computing Euler numbers in just one single raster scan of the image. A modification of this algorithm that can be run in a Field Programmable Gate Array with a pipelined architecture as completely described in [10].

In [11], Euler numbers are used to analyze textural and topologial feaures of signature images, while in [12], this same feature is used to describe the structural effects caused by noise in binary images. In short, in [13], the Euler characteristic is used to extract lung regions from gray level chest $\mathrm{x}$-ray images.

In [14], the authors present a fast algorithm for computing the Euler number of an image and its VLSI implementation, while in [15], the authors describe the functioning of a pipeline architecture for performing the same task. In short, in [16], an on-chip computation of Euler number of a binary image for efficient database search is presented.

A first patent to compute the Euler number of a binary image was introduced by Acharya et al in [17].

Other methods have been reported in literature to compute the Euler number of a binary image. Refer for example to [18-26]. In [18] the Euler characteristic is obtained by means of a quadtree representation of the image being analyzed. In [19], linear quadtrees are used to perform the same task, while in [20] a bintree representation is used. In [21] the Euler number is considered as a value of certain additive functional belonging to the so-called quermass-integrals family, while in [22] it is obtained in terms of the vertices, basic square faces and edges of the square graph of a binary image. In [23] it is computed by means of the socalled connectivity graph of the image. In [24] an integral geometric approach is used to compute the Euler feature for spatial images. A proof of an Euler number equation is given in [25]. In short, in [26], the Euler number of a binary image is computed in terms of the number of terminal points (points with just one neighbour) and the number of three-edge-points (points with only three neighbours) of the skeletons of regions inside the image. If Tps is the number of terminal points of $n$ skeletons from $\mathrm{n}$ regions in the image and TEps is 
the number of three-edge-points of these $n$ skeletons, then:

$$
E=\frac{T p s-T E p s}{2}
$$

In the case of the images shown in Fig. 1 (b): Tps (left image) $=5$ and TEps (left image) $=3$, thus $E$ (left image $)=1$. Similarly: Tps (right image) $=3$ and TEps (right image) $=3$, thus $E$ (right image) $=$ 0 .

With the exception of the method reported in [27], all the other approaches reported in [18-26] have been designed to work with images composed of squared cells. In [27], morphological operations are used to compute the Euler number of a binary image composed of hexagonal cells.

Taking the above discussion into account, in this paper, a completely different approach to compute the Euler number of a binary image composed of hexagonal cells is described. Images composed of hexagonal configurations, as we shall see later, offer advantages over images composed of square or triangular cells.

The rest of this paper is organized as follows. In section 2 , the proposal is fully described. In section 3 , several examples to illustrate the functioning and efficiency of the method are provided. In section 4 a sketch of an algorithmic procedure is given. Finally, section 5 is focussed on the conclusions and directions for further work.

\section{The proposal}

In this section the proposal to compute the Euler number of a binary image (or binary region) composed of hexagonal cells is described. For this, the concepts of perimeter: $P$ and contact perimeter: $P_{c}$ are given first. Hereafter, these definitions are valid for triangular, rectangular and hexagonal cells.

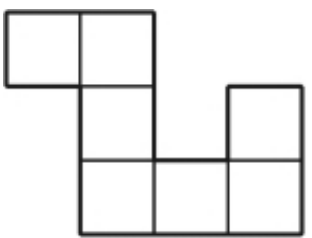

(a)

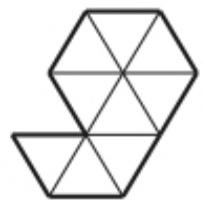

(c)

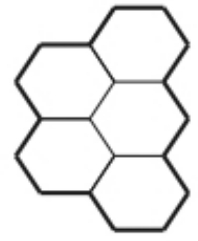

(e)

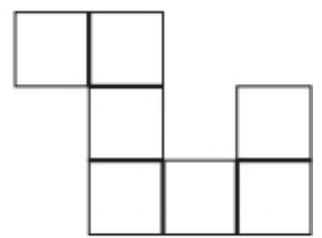

(b)

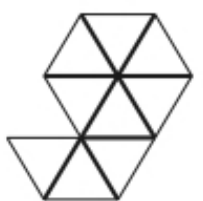

(d)

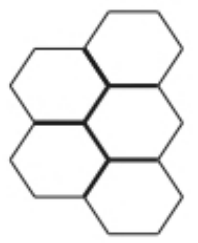

(f)
Figure 2. Perimeter and contact perimeter of three simples binary shapes.

According to [28], the perimeter $\mathrm{P}$ of the shapes (regions) in an image composed by cells (pixels) corresponds to the sum of lengths of the exterior sides of each shape. The exterior sides of a shape are also sides of background pixels. The exterior sides of the shapes in Figures 2(a), 2(c) and 2(e), are shown in bold. Thus, the values of the perimeter of the shapes shown in Figures 2(a), 2(c) and 2(e) are 16, 9, and 16, respectively. From the same reference [28], the contact perimeter of a region from an image composed of cells corresponds to the sum of the lengths of segments that are common to two cells. For example, the contact perimeter of the shapes shown in Figures 2(b), 2(d) and 2(f) equals 6, 9 and 7, respectively. Based on these two parameters, in [28], the authors show that the following relationship holds: For any shape $\mathrm{Sn}$, composed of $\mathrm{n}$ cells, it holds that: 


$$
2 P_{c}+P=n T
$$

where again $P_{c}$ is the contact perimeter, $P$ is the perimeter of the shape and $T$ is the number of sides of the cell. For a triangular cell, $T=3$, for a square cell, $T=4$ and for a hexagonal cell, $T=6$.

Although the theory that we are going to present in the following paragraphs is valid for regions composed of squared, triangular and hexagonal cells (even in the case of three-dimensional cells, with some modifications), in this paper we are going to deal only with images composed of hexagonal cells (cells for which, $T=6$ ). The main reason for this is that regions composed of hexagonal cells (Figure 3(a)) do not suffer from the problems of connectivity frequently found in the case of regions composed of squared or triangular cells. In the case of hexagonal cells, they will never appear connected by their corners, as can happen with squared or triangular cells. In the particular case of squared cells, for example, if 8-connectivity is chosen, the five square pixels shown in Figure 3(b) will form a whole region, however if 4connectivity is chosen, then we will have two regions. Something similar can be said in the case of the six triangular cells shown in Fig. 3(c).

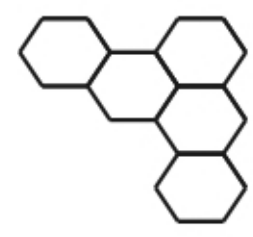

(a)

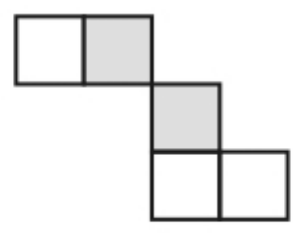

(b)

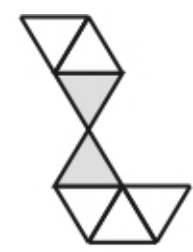

(c)
Figure 3. Images composed of hexagonal cells do not present the connectivity problems, sometimes associated with images composed of squares or triangles. As can be seen from this figure (cases (b) and (c)), cells might appear connected by their corners.

Before providing a formula to compute the Euler number of a binary image composed of a set of connected regions $(T=6)$, the concepts of a redundant cell and the number of times a cell is redundant have to be first defined. For this, let us consider the 6-neighbourhood of a pixel $p$ (Figure.4)

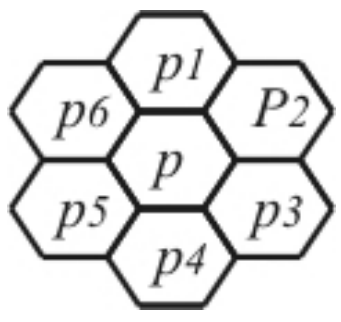

Figure 4. Neighborhood of pixel $p$.

Definition 1. In the case of hexagonal region-cells, if $N(p)$ and $N T(p)$ are, respectively, the number of 1-pixels around $p$ and the number of 0 to 1 transitions in the sequence $P 1, P 2, P 3, P 4, P 5, P 6$, then cell $p$ is called redundant if inside its neighbourhood satisfies the following two conditions:

1. $N(p) \geq 2$.

2. $N(p)-N T(p) \geq 1$.

According to Definition 1, in Figures 5(a) and 5(b) $p$ is redundant, while in Figures 5(c) and 5(d), it is not.

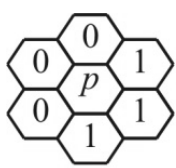

(a)

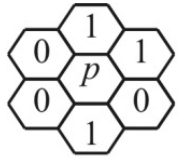

(b)

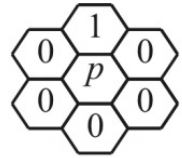

(c)

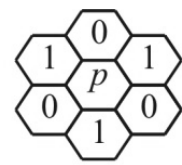

(d)
Figure 5. Fig. 5. Examples of arrangements where $p$ is redundant or not. (a) $p$ is redundant

$$
\begin{gathered}
\text { because } N(p)=3 \text { and } \\
N(p)-N T(p)=3-1=2 \geq 1 \text {. (b) } p \text { is } \\
\text { redundant considering that } N(p)=3 \text { and } \\
N(p)-N T(p)=3-2=1 \geq 1 \text {. (c) } p \text { is not } \\
\text { redundant because } N(p)=1 \text { and } \\
N(p)-N T(p)=1-1=0<1 \text { and (d) } p \text { is not } \\
\text { redundant taking into account that } N(p)=3 \\
\text { but } N(p)-N T(p)=3-3=0<1 .
\end{gathered}
$$


Definition 2. In the case of hexagonal region-cells, if $N(p)$ and $N T(p)$ are, respectively, the number of 1 -pixels around $p$ and the number of 0 to 1 transitions in the sequence $P 1, P 2, P 3, P 4, P 5, P 6$, the number of times a cell $p$ is redundant inside its neighbourhood it given as:

$$
N(p)-N T(p)
$$

Again, from Figure 5(a) $\mathrm{p}$ is redundant twice for $N(p)-N T(p)=3-1=2$, while in Figure $5(\mathrm{~b})$ $\mathrm{p}$ is redundant once for $N(p)-N T(p)=3-2=1$.

Let $N R$ be the number of times all the cells in an image are redundant.

We are now in the position to establish the relation that governs the connectivity of the hexagonal pixels of shapes of a binary image that allows computing the Euler number of this image.

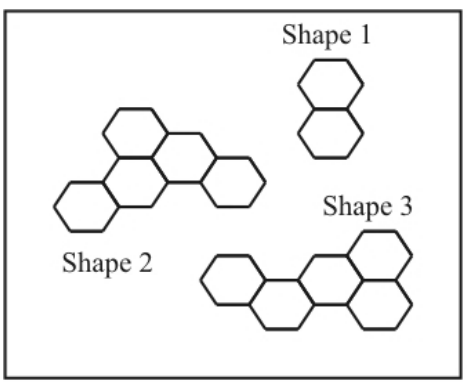

(a)

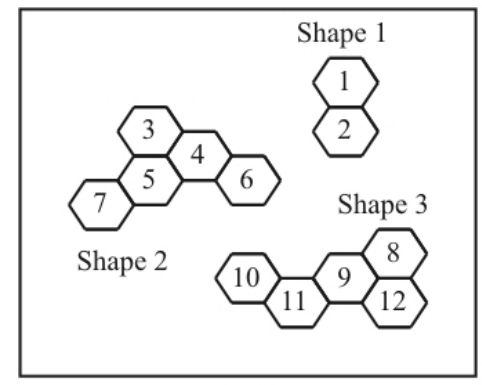

(c)
Proposition 1. For any binary image composed of hexagonal cells $(T=6)$, its Euler number is given as follows:

$$
E=\frac{P-(T-2) P_{c}+2 \cdot N R}{T}
$$

Where, as mentioned before $P$ and $P_{c}$ are, respectively, the perimeter and contact perimeter of the shape, $N R$ is the number of times all the cells in an image are redundant, and $T$ is the number of sides of the cell.

Proof. See the annex.

\section{Example}

To numerically verify the correct functioning of equation (5), in this section we present several numerical examples. For this, the two images shown in Fig. 6(a) and 6(b), respectively, are used. As can be seen from this figure, the first image is composed of three connected regions, while the second image is composed of two connected regions.

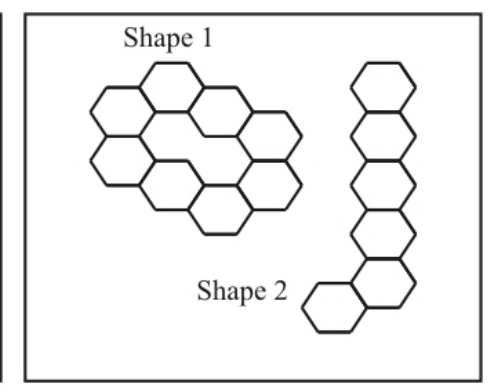

(b)

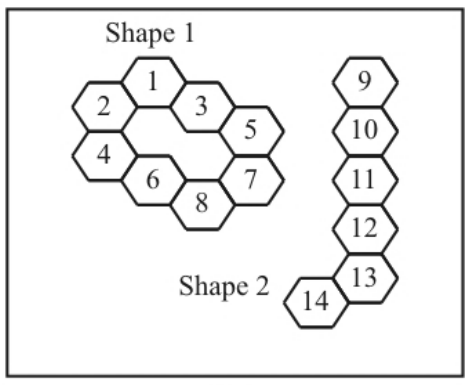

(d)

Figure 6. (a) A binary image containing three different connected components. (b) A binary image containing two different connected components, one of them containing a hole. (c) and (d) Same images as in (a) and (b) but with labelled pixels. 
For each shape in the images shown in Fig. 6(a) and 6(b), Tables 1 and 2 summarize the computation results for the Euler number $E$ of these images.

The reader can easily verify the validity of these results by using the standard equation given by (1).

In the next section we present a computational procedure to compute the Euler number of a binary shape in terms of equation 5 .

\section{Example}

In this section, we describe a procedure to compute the Euler number of the image. It is not difficult to see that the Euler number of a binary image can be computed either in series or in parallel. From now on, let us focus on the parallel way.

The partial calculation of $E$ can be thus computed by evaluating the contributions to the values of $P$,
$P_{c}$, and $N R$ for each $p=1$ of the image. To show how this is possible, in the case of $P, P_{c}$ and $N R$, let us take into account again, the neighbourhood shown in Fig. 4. Thus for each pixel $p$ in the image with value 1 :

Compute the value of $P$ by counting the number of 6 -neighbors with value 0 around $p$. For example, for pixel $p$ of Fig. 7(b), $P=3$, while for pixel $p$ of Fig. 7(c), $P=2$.

Compute the value of $P_{c}$ by counting the number of 6 -neighbors with value 1 around $p$. Thus, for example, for pixel $p$ (Fig. 7(b), $P_{c}=3$, while for pixel $p$ of Fig. 7(c), $P_{c}=4$. From these two examples we notice that for a pixel $p(p=1)$, $P+P_{c}=T=6$. So, instead of counting the number of 6-neighbors with value 1 around pixel $p$, $P_{c}$ can be directly computed as $P_{c}=6-P$.

\begin{tabular}{|c|c|c|}
\hline Shape 1: & Shape 2: & Shape 3: \\
\hline$P_{1}=10, P C_{1}=1, N R=0$. & $P_{2}=20, P C_{2}=5, N R=3$. & $P_{2}=20, P C_{2}=5, N R=3$. \\
\hline \multicolumn{3}{|c|}{$P_{\text {total }}=P_{1}+P_{2}+P_{3}=50$} \\
\hline \multicolumn{3}{|c|}{$P c_{\text {total }}=P c_{1}+P c_{2}+P c_{3}=11$} \\
\hline \multicolumn{3}{|c|}{$N R_{\text {total }}=N R_{1}+N R_{2}+N R_{3}=6$} \\
\hline \multicolumn{3}{|c|}{$E=\frac{50-(4) 11+2 \cdot 6}{=} \underline{50-44+12}=\frac{18}{=3 .}$} \\
\hline & $6=\frac{6}{6}$ & \\
\hline
\end{tabular}

Table 1. Computation results for the three shapes of Fig. 6(a).

\begin{tabular}{|c|c|}
\hline Shape 1: & Shape 2: \\
\hline$P_{1}=32, P C_{1}=8, N R=0$. & $P_{2}=26, P C_{2}=5, N R=0$ \\
\hline \multicolumn{2}{|c|}{$P_{\text {total }}=P_{1}+P_{2}=58$} \\
\hline \multicolumn{2}{|c|}{$P c_{\text {total }}=P c_{1}+P c_{2}=13$} \\
\hline \multicolumn{2}{|c|}{$N R_{\text {total }}=N R_{1}+N R_{2}=0}$. \\
\hline & $=1$ \\
\hline
\end{tabular}

Table 2. Computation results for the two shapes of Fig. 6(b). 
Determine if $p$ is redundant, and if it is, compute the number of times $p$ is redundant inside its neighbourhood. This can be done by applying equation (4) to pixel $p$. For pixel $p$ of Figure $7(b)$ $N(p)-N T(p)=3-2=1$, thus, in this case, $p$ is redundant once, while for pixel $p$ of Figure $7(\mathrm{c})$ $N(p)-N T(p)=4-2=2$, thus $p$ is redundant twice.

Is short, for pixel $p$ from Figure $7(\mathrm{~b}), P=3, P_{c}=3$ and $N R=1$, while for pixel $p$ of Figure $7(\mathrm{c}), P=2$, $P_{c}=4$ and $N R=2$.

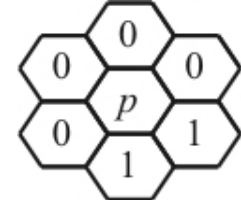

(a)

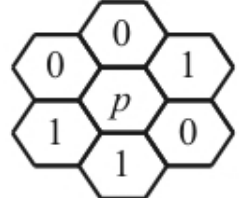

(b)

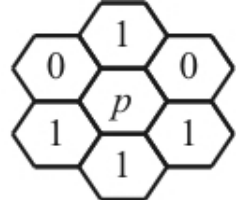

(c)
Figure 7. (a) Pixel $p$ has values for $P=4, P_{c}=2$ and $N R=1$. (b) Pixel $p$ has values for $P=3$, $P_{c}=3$ and $N R=1$. (c) Pixel $p$ has values for $P=2, P_{c}=4$ and $N R=2$.
From the above discussion we are ready to provide a procedure to compute the Euler number of a binary image composed of hexagonal cells.

Procedure: Computation of Euler number $E$ of a binary image with hexagonal pixels:

Input: A binary image possibly containing one or more connected regions with and without holes.

1: Initialize $P=P_{c}=N R=0$.

2: For each pixel $p=1$ in the image:

3: Compute the increment of $P(\Delta P)$ by counting the number of 0 s in its neighbourhood (see Fig. 4). Compute the new value of $P \quad\left(P_{\text {new }}\right)$ as $P_{\text {new }}=P_{\text {old }}-\Delta P$.

4: Compute the increment of $P_{c}\left(\Delta P_{c}\right)$ as $P_{c}=6-P$. Compute the new value of $P_{c}\left(P_{c_{\text {new }}}\right)$ as $P_{c_{\text {new }}}=P_{c_{\text {old }}}-\Delta P_{c}$.

5: Compute the increment of $N R(\Delta N R)$ as $N(p)-N T(p)$. Compute the new value of $N R$ $\left(N R_{\text {new }}\right)$ as $N R_{\text {new }}=N R_{\text {old }}-\Delta N R$.

6: end for

\begin{tabular}{|c|c|c|c|c|c|c|c|c|c|c|c|}
\hline \multicolumn{4}{|c|}{ Shape 1: } & \multicolumn{5}{c|}{ Shape 2: } & \multicolumn{4}{c|}{ Shape 3: } \\
\hline $\begin{array}{c}\text { Píxel } \\
\text { No. }\end{array}$ & $P$ & $P_{c}$ & $N R$ & $\begin{array}{c}\text { Píxel } \\
\text { No. }\end{array}$ & $P$ & $P_{c}$ & $N R$ & $\begin{array}{c}\text { Píxel } \\
\text { No. }\end{array}$ & $P$ & $P_{c}$ & NR \\
\hline 1 & 5 & 1 & 0 & 3 & 4 & 2 & 1 & 8 & 4 & 2 & 1 \\
\hline 2 & 5 & 1 & 0 & 4 & 3 & 3 & 1 & 9 & 3 & 3 & 1 \\
\hline & & & & 5 & 3 & 3 & 1 & 10 & 5 & 1 & 0 \\
\hline & & & & 6 & 5 & 1 & 0 & 11 & 4 & 2 & 0 \\
\hline & & & & 7 & 5 & 1 & 0 & 12 & 4 & 2 & 1 \\
\hline- & 10 & 2 & 0 & - & 20 & 10 & 3 & - & 20 & 10 & 3 \\
\hline
\end{tabular}

Table 3. Computation results for each of the three shapes of binary image shown in Fig. 6(c) by means of the described procedure. The last row of this table contains the total values

of $P, P_{c}$ and $N R$ for each of the three shapes 1,2 and 3 . 
The reader can easily verify that at the end of this procedure, we will have the value of $P_{c}$ doubled. To get the correct value of this parameter, it would e thus necessary to divide the obtained value by two.

For the example given in section 3, Tables 3 to 6 summarize the computation results pixel by pixel of each shape of Figures $6(a)$ and $6(b)$. For this the labelled pixels shown in Figures 6(c) and 6(d) were used.
From Table 4, as mentioned before, we note that the value for $P_{c}$ is double the expected value. Before applying equation 5 , the value for $P_{c}$ should be divided twice. Thus $P_{c}=22 / 2=11$. Because from this table, $P=50$ and $N R=6$, then:

$$
E=\frac{50-(6-2) 11+2 \cdot 6}{6}=\frac{62-44}{6}=\frac{18}{6}=3 .
$$

Again, the reader can easily verify that this is the correct value for $E$ from this example.

\begin{tabular}{|c|c|c|}
\hline \multicolumn{3}{|c|}{ Total values for the image } \\
\hline$P$ & $P_{c}$ & $N R$ \\
\hline $50=10+20+10$ & $22=2+10+10$ & $6=0+3+3$ \\
\hline
\end{tabular}

Table 4. Total computation results for the three shapes of binary image shown in Fig. 6 (c) by means of the described procedure.

\begin{tabular}{|c|c|c|c|c|c|c|c|}
\hline \multicolumn{4}{|c|}{ Shape 1: } & \multicolumn{4}{c|}{ Shape 2: } \\
\hline $\begin{array}{c}\text { Píxel } \\
\text { No. }\end{array}$ & $P$ & $P_{c}$ & $N R$ & $\begin{array}{c}\text { Píxel } \\
\text { No. }\end{array}$ & $P$ & $P_{c}$ & $N R$ \\
\hline 1 & 4 & 2 & 0 & 9 & 5 & 1 & 0 \\
\hline 2 & 4 & 2 & 0 & 10 & 4 & 2 & 0 \\
\hline 3 & 4 & 2 & 0 & 11 & 4 & 2 & 0 \\
\hline 4 & 4 & 2 & 0 & 12 & 4 & 2 & 0 \\
\hline 5 & 4 & 2 & 0 & 13 & 4 & 2 & 0 \\
\hline 6 & 4 & 2 & 0 & 14 & 5 & 1 & 0 \\
\hline 7 & 4 & 2 & 0 & & & & \\
\hline 8 & 4 & 2 & 0 & & & & \\
\hline- & 32 & 16 & 0 & - & 26 & 10 & 0 \\
\hline
\end{tabular}

Table 5. Computation results for each of the three shapes of binary image shown in Fig. 6(d) by means of the described procedure. The last row of this table contains the total values of $P, P_{c}$ and $N R$ for each of the two shapes 1 and 2. 


\begin{tabular}{|c|c|c|}
\hline \multicolumn{3}{|c|}{ Total values for the image } \\
\hline$P$ & $P_{c}$ & NR \\
\hline $58=32+26$ & $26=16+10$ & $0=0+0$ \\
\hline
\end{tabular}

Table 6. Total computation results for the two shapes of the binary image shown in Fig. 6(d) by means of the described procedure.

\section{Discussion}

From these two examples, it can be seen that the proposed new equation and the algorithmic procedure described in this section, shows that the Euler feature of a binary image composed of shapes formed of hexagonal cells can be computed. Also as the partial results are independent from pixel to pixel, the proposal can be applied in parallel, allowing us to speed up the computing procedure.

When applying the described algorithmic procedure, the user has to take into account the fact that the value of $P_{c}$ will be double the expected value. So, before applying equation 5 , the value of $P_{c}$ should be divided twice.

\section{Conclusions}

In this paper a new proposal to compute the Euler number or genus of a binary image composed of hexagonal cells is described. The computation of this important feature is based on information directly obtained from the cells of the shapes in the image: the perimeter, the contact perimeter and the number of times a cell is redundant.

The main features of this proposal are its simplicity and originality. As shown, the computation of the Euler number in this case can be applied in parallel. The implementation of the procedure in a FPGA will certainly allow computing the Euler number of a binary image in real time applications.

With investigations in the development of new hexagonal-cell-based CCD technologies, certainly algorithms such as reported in this paper, will in the near future find many applications that can be more efficiently handled than with standard rectangular-cell-based CCD sensors.

At the moment we are adapting this proposal to work in the case of squared and triangular cells. Also we are extending it to be applied in the case of cubical cells.

\section{References}

[1] X. He and W. Jia (2005). Hexagonal Structure for Intelligent Vision, Information and Communication Technologies, ICICT, pp. 52- 64 .

[2] R. C. Staunton (1989). The design of hexagonal sampling structures for image digitization and their use with local operators, Image Vision Computing, 7(3):162166.

[3] L. Middleton and J. Sivaswamy (2005). Hexagonal Image Processing; A Practical Approach, Springer.

[4] L. Middleton and J. Sivaswamy (2001). Edge Detection in a Hexagonal-Image Processing Framework. Image and Vision Computing 19:1071-1081.

[5] A. Rosenfeld and A. C. Kak (1982). Digital Picture Processing, Academic Press Inc. New York.

[6] R. C. Gonzalez and R. E. Woods (1993). Digital Image Processing, Addison-Wesley, Reading, Massachussets.

[7] S. D. Zenzo, L. Cinque and S. Levivaldi (1996). Runbased algorithms for binary image analysis and processing, IEEE Transactions on PAMI, 18(1):83-89.

[8] H. S. Yang and S. Sengupta (1988). Intelligent shape recognition for complex industrial tasks, IEEE Control Systems Magazine 8(3):23-29. 
[9] L. Snidaro and G. L. Foresti (2003). Real-time Thresholding with Euler Numbers. Pattern Recognition Letters 24(9-10):1533-1544.

[10] J. Athow, N. Abbasi and A. Amer (2009). A RealTime FPGA Architecture of a Modified Stable EulerNumber Algorithm for Image Binarization. Technical Report 2009-1-ATHOW Department of Electrical and Computer Engineering, Concordia University. January 2009.

[11] M. Vatsa, R, Singh, P. Mitra and A. Noore (2004). Signature Verification using Static and Dynamic Features (2004). ICONIP 2004. LNCS 3316. SpringerVerlag, Pp. 350-355.

[12] Ch. Zhang, Z. Qiu and D. Sun and J. Wu (2006). Euclidean Quality Assessment for Binary Images. 18th International Conference on Pattern Recognition, ICPR 2006. Pp. 300-303.

[13] L. P. Wong and H. T. Ewe (2007). A Sutdy of Nodule Detection using Opaque Object Filter. Biomed 06. IFMBE Proceedings 15. Pp. 236-240. Springer Verlag.

[14] S. Dey, B.B. Bhattacharya, M.K. Kundu, T. Acharya (2000). A fast algorithm for computing the Euler number of an image and its VLSI implementation, in Proc. 13th International Conference on VLSI Design, pp. 330-335.

[15] A. Bishnu, B. B. Bhattacharya, M. K. Kundu, C.A. Murthy, T. Acharya (2005). A pipeline architecture for computing the Euler number of a binary image. Journal of Systems Architecture 51(8):47-487.

[16] A. Bishnu, B. B. Bhattacharya, M. K. Kundu, C.A. Murthy, T. Acharya (2001). On chip computation of Euler number of a binary image for efficient database search, Proc. of the International Conference on Image Processing (ICIP), Vol. III, pp. 310-313.

[17] T. Acharya, B. B. Bhattacharya, A. Bishnu, M. K. Kundu, Ch. A. Murthy (2006). Computing the Euler Number of a Binary Image. United States Patent 7027649 B1. April 11, 2006.

[18] Ch. R. Dyer (1980). Computing the Euler number of an image from its quatree. Comput. Vision, Graphics Image Process. 13, 270-276.

[19] H. Samet et al. (1985). Computing Geometric Properties of Images Represented by Linear Quadtrees. IEEE Trans. PAMI, 7(2):229-240.
[20] H. Beri (1987). Computing the Euler characteristic and related additive functionals of digital objects from their beentree representation, Comput. Vision, Graphics Image Process. 40, 115-126.

[21] H. Beri and W. Nef (1984). Algorithms for the Euler characteristic and related additive functionals of digital objects, Comput. Vision, Graphics Image Process. 28, 166-175.

[22] M. H. Chen and P. F. Yan (1988). A fast algorithm to calculate the Euler number for binary images, Pattern Recognition Letters 8(12):295-297.

[23] F. Chiavetta and V. Di Gesú (1993). Parallel computation of the Euler number via connectivity graph, Pattern Recognition Letters 14(11):849-859.

[24] W. Nagel, J. Ohser and K. Pischang (2000). An integral-geometric approach for the Euler-Poincare characteristic of spatial images. Journal of Microsc, 189:54-62.

[25] LIN Xiaozhu, SHA Yun, JI Junwei \& WANG Yanmin (2006). A proof of image Euler Number formula. Science in China: Series F Information Sciences 49(3):364-371.

[26] J. L. Díaz de León S. and H. Sossa (1996). On the computation of the Euler number of a binary object. Pattern Recognition, 29(3):471-476.

[27] J. Serra (1982). Image Analysis and Mathematical Morphology, Academic Press.

[28] E. Bribiesca (1997). Measuring 2-D shape compactness using the contact perimeter, Computers Math. Applic. 33(11):1-9.

\section{Acknowledgments}

The authors wish to thank SIP-IPN for the economical support under grant number 20100468. The authors also thank the European Union, the European Commission and CONACYT for their economical support. This paper has been prepared by economical support of the European Commission under grant FONCICYT 93829. The content of this paper is an exclusive responsibility of the IPN and the UDEG and it cannot be considered to reflect the position of the European Union. We also thank the reviewers for their comments to improve this paper. 


\section{Computation of the Euler Number of a Binary Image Composed of Hexagonal Cells, J. H. Sossa-Azuela et al., 340-351}

\section{Annex}

\section{Proof of Proposition 1.}

Proof. Instead of an exhaustive proof of proposition 1, we provide an intuitive proof. For the base case, and without lost of generality we suppose that the image contains one shape composed of just one cell as shown in Figure 8(a). In this case we have that $P=T, P_{c}=0$ and $N R=0$, thus by direct calculation $E=T / T=1$.

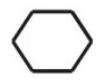

(a)

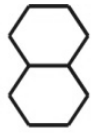

(b)

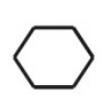

(c)
Fig. 8. (a) Initial shape composed of one base cell. (b) First case: The additional cell is connected to the initial cell by one of its sides. (c) Second case: The additional cell is not connected to the initial cell.

Now, let $S_{n+1}$ be the shape composed of the initial shape $S_{n}$ plus any cell. Let $L$ be the number of contact sides of this new cell and $P_{c}^{\prime}$ and $P^{\prime}$ be the corresponding perimeters of $S_{n+1}$, thus:

$$
\begin{gathered}
P_{c}^{\prime}=P_{c}+L \\
P^{\prime}=P-L+(T-L)=P+T-2 L
\end{gathered}
$$

This means that for the new shape $S_{n+1}$ the contact perimeter is increased by $L$ while the perimeter is decreased by $L$ and increased by $(T-L)$. This corresponds to the contribution of the new cell to the perimeter. Let also NR' be the number of redundant cells added to the original shape.

Now if $E^{\prime}$ is the new value for the new shape $S_{n+1}$, and because no increment to $N R^{\prime}$ occurs $\left(N R^{\prime}=N R\right)$, then:

$$
\begin{aligned}
E^{\prime} & =\frac{P^{\prime}-(T-2) P_{c}{ }^{\prime}+2 N R^{\prime}}{T}=\frac{P+T-2 L-(T-2)\left(P_{c}+L\right)+2 N R}{T} \\
& =\frac{P-(T-2) P_{c}+2 N R+T(1-L)}{T}=E+1-L .
\end{aligned}
$$

As can be seen from the new shape $S_{n+1}$, the value of $E$ is increased by 1 and decreased by $L$.
Now let us study the two possible cases:

Case 1: The added cell is connected to the initial cell by one of its sides (for a possible arrangement, refer to Fig. 8 (b)).

In this case we should have: $E^{\prime}=E$.

In this case $L=1$, thus $E^{\prime}=E+1-1=E$

Case 2: The added cell is not connected to the initial cell (for an example, refer to Fig. 6 (c):

In this case we should have: $E^{\prime}=E+1$.

In this particular case $L=0$, thus

$E^{\prime}=E+1-0=E+1$ 


\section{Authors' Biographies}

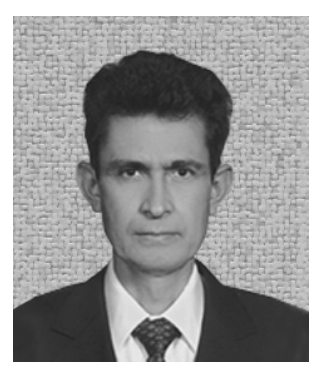

\section{Humberto SOSSA-AZUELA}

He received the B.S. degree in Electronics and Communications Engineering from the University of Guadalajara, Mexico in 1980, the M.Sc. degree in Electrical Engineering from CINVESTAV-IPN, Mexico in 1987, and the Ph.D. degree from National Polytechnic Institute of Grenoble, France in 1992. From 1997 he has been with National Polytechnic Institute of Mexico, where he is currently a full-time Professor in the Computing Research Center. From 1988, he is also a member of the Mexican National Research System (SNI). His research interest includes image analysis, pattern recognition, neural networks applied to image retrieval, shape description and robot control.

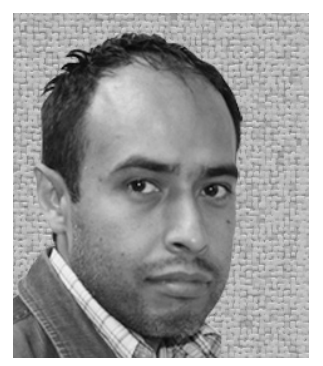

\section{Erik CUEVAS-JIMÉNEZ}

$\mathrm{He}$ received the B.S. degree with distinction in Electronics and Communications Engineering from the University of Guadalajara, Mexico in 1995, the M.Sc. degree in Industrial Electronics from ITESO, Mexico in 2000, and the Ph.D. degree from Freie Universität Berlin, Germany in 2005. Since 2001 he was awarded a scholarship from the German Service for Academic Interchange (DAAD) as full-time researcher. From 2006 he has been with University of Guadalajara, where he is currently a full-time Professor in the Department of Computer Science. From 2008, he is also a member of the Mexican National Research System (SNI). His research interest includes computer vision and artificial intelligence applications in medical image.

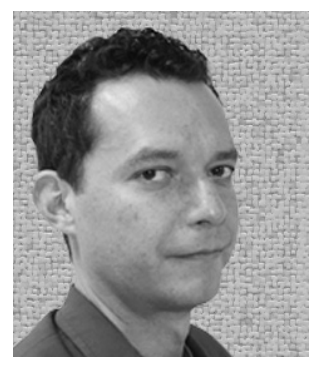

\section{Daniel ZALDIVAR-NAVARRO}

He received the B.S. degree with distinction in Electronics and Communications Engineering from the University of Guadalajara, Mexico in 1995, the M.Sc. degree in Industrial Electronics from ITESO, Mexico in 2000, and the Ph.D. degree from Freie Universität Berlin, Germany in 2005. From 2001 he was awarded a scholarship from the German Service for Academic Interchange (DAAD) as full-time researcher. From 2006 he has been with University of Guadalajara, where he is currently a Professor in the Department of Computer Science. From 2008 he is also a member of the Mexican National Research System (SNI). His current research interest includes biped robots design, humanoid walking control, and artificial vision. 